\title{
A Novel Way Of Taking Trauma Radiographs Of A Stretcher Patient In A Field Hospital
}

\author{
DJ Vassallo, PC Rowlands
}

Lt Col DJ Vassallo

RAMC,

Consultant General

Surgeon

Email: DJVassallo@aol.com

Sgt PC Rowlands RAMC

Combat Medical

Technician

34 Field Hospital

Troop, OP FINGAL, BFPO 758 .

\section{Introduction}

A common challenge facing resuscitation staff in a field hospital environment is how best to take radiographs of trauma patients lying on field stretchers. The authors describe a simple method using existing stretcher fittings that should greatly simplify radiography in the field.

\section{Methods}

The new general issue field stretcher incorporates three body straps to hold the patient in place during transport. Two of these straps can be unclipped on arrival of the patient in Resus, passed under the stretcher and refastened, thereby forming two slings to support the $\mathrm{X}$-ray cassette. The position of the straps can be easily adjusted by sliding their attachment along the rail in the stretcher poles.

Figure 1 shows a single strap supporting the X-ray cassette in position under the patient's chest, in conjunction with the support provided by the top stretcher bar. The upper clamp on the hospital chassis has to be loosened first to allow the cassette to pass over the bar.

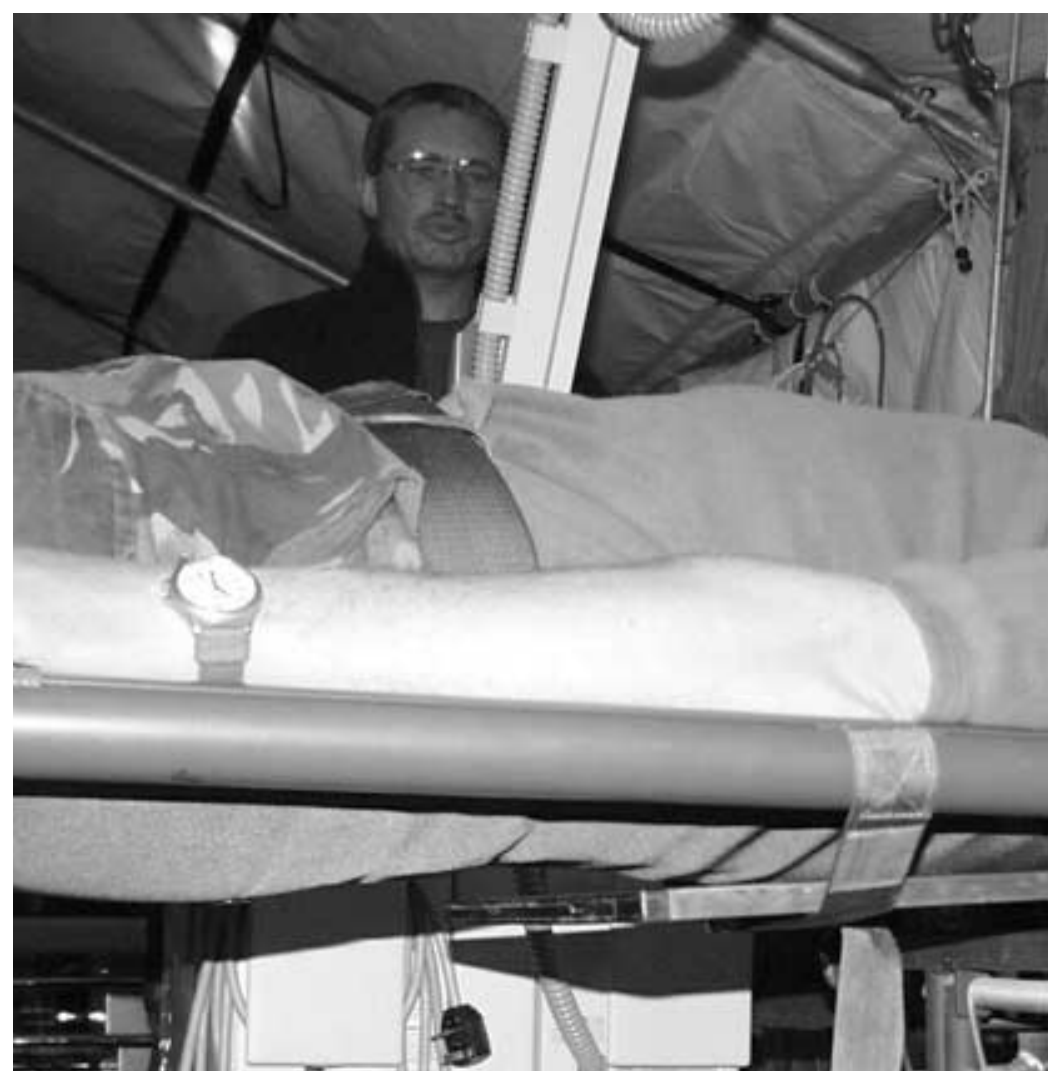

Fig 1. cassette strapped in place for chest radiograph.
Figure 2 shows two straps supporting the $\mathrm{X}$-ray cassette in position under the patient's pelvis.

If the stretcher is placed on trestles as opposed to a hospital chassis, the trestle at the head end has to be positioned as shown in Figure 3 to allow the X-ray cassette to be placed into the correct position for a chest radiograph.

\section{Discussion}

The normal trauma X-ray series consists of antero-posterior chest and pelvic radiographs, followed by a lateral cervical spine radiograph.

Hitherto, patients lying on field stretchers have had to be bodily manhandled in order to fit the X-ray cassette under the patient's chest, with care being taken to maintain inline cervical spine immobilisation. The patient has to be lifted again to remove the cassette. These manoeuvres have to be repeated to obtain pelvic views.

In a trauma scenario, this may cause undue pain and distress to the patient, the cervical spine is potentially at risk, and the movement may exacerbate internal haemorrhage. It requires at least two attending staff to bodily lift the patient whilst another member of staff protects the cervical spine and the radiographer inserts the cassette between patient and stretcher. The cassette is at risk of contamination by blood or body fluids. Attending staff have to interrupt all other clinical procedures during this process.

The simple method described, which can also be used for lower limb radiographs, averts all these problems. The patient does not have to be moved at all, the cervical spine remains fully immobilised, attending staff do not have to interrupt other procedures, and the cassette can be placed in position with ease by the radiographer.

\section{Acknowledgements}

To SSgt P Parker, radiographer at $34 \mathrm{Fd}$ Hosp Tp, for his assistance. 


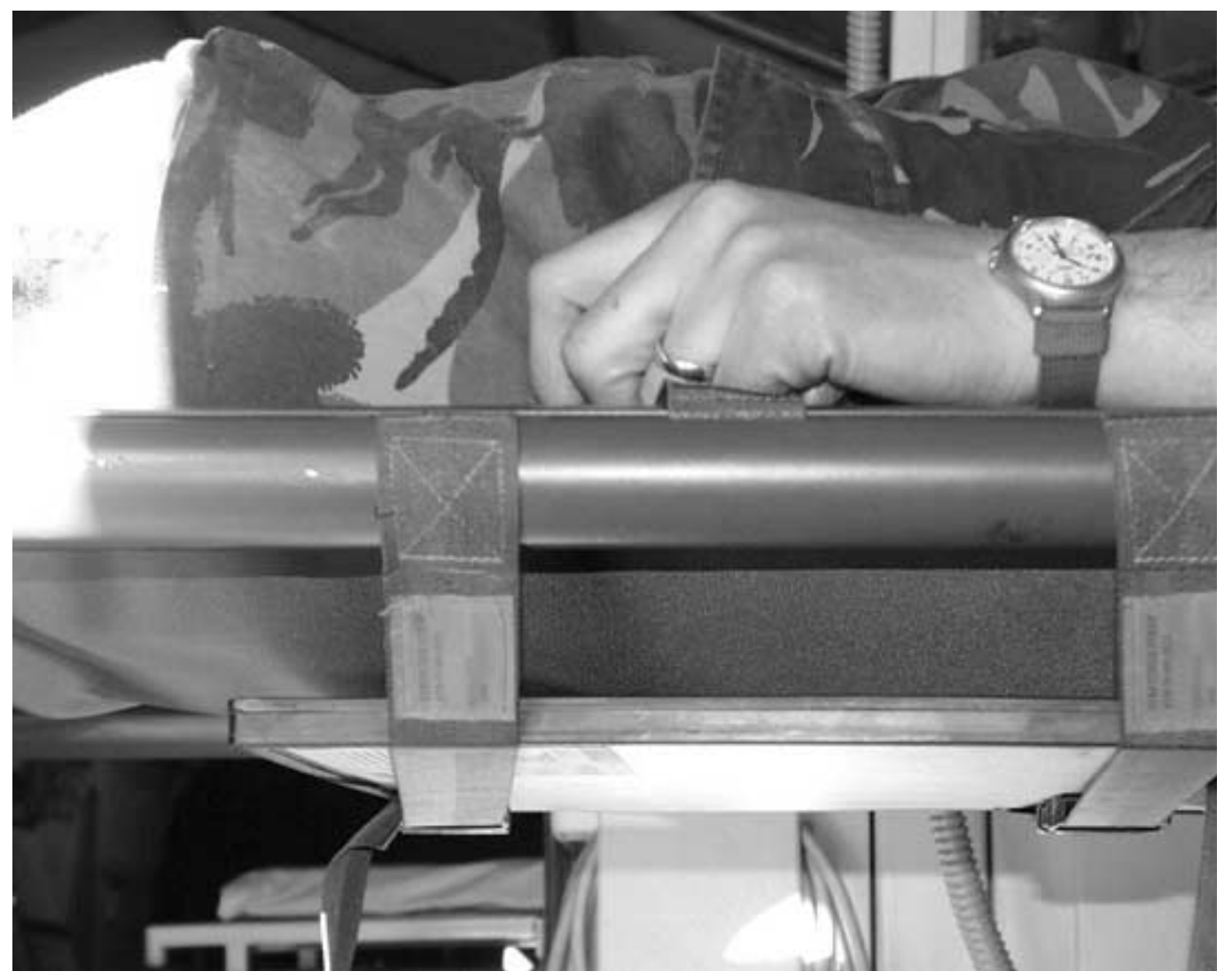

Fig 2. pelvic $x$-ray cassette in place.

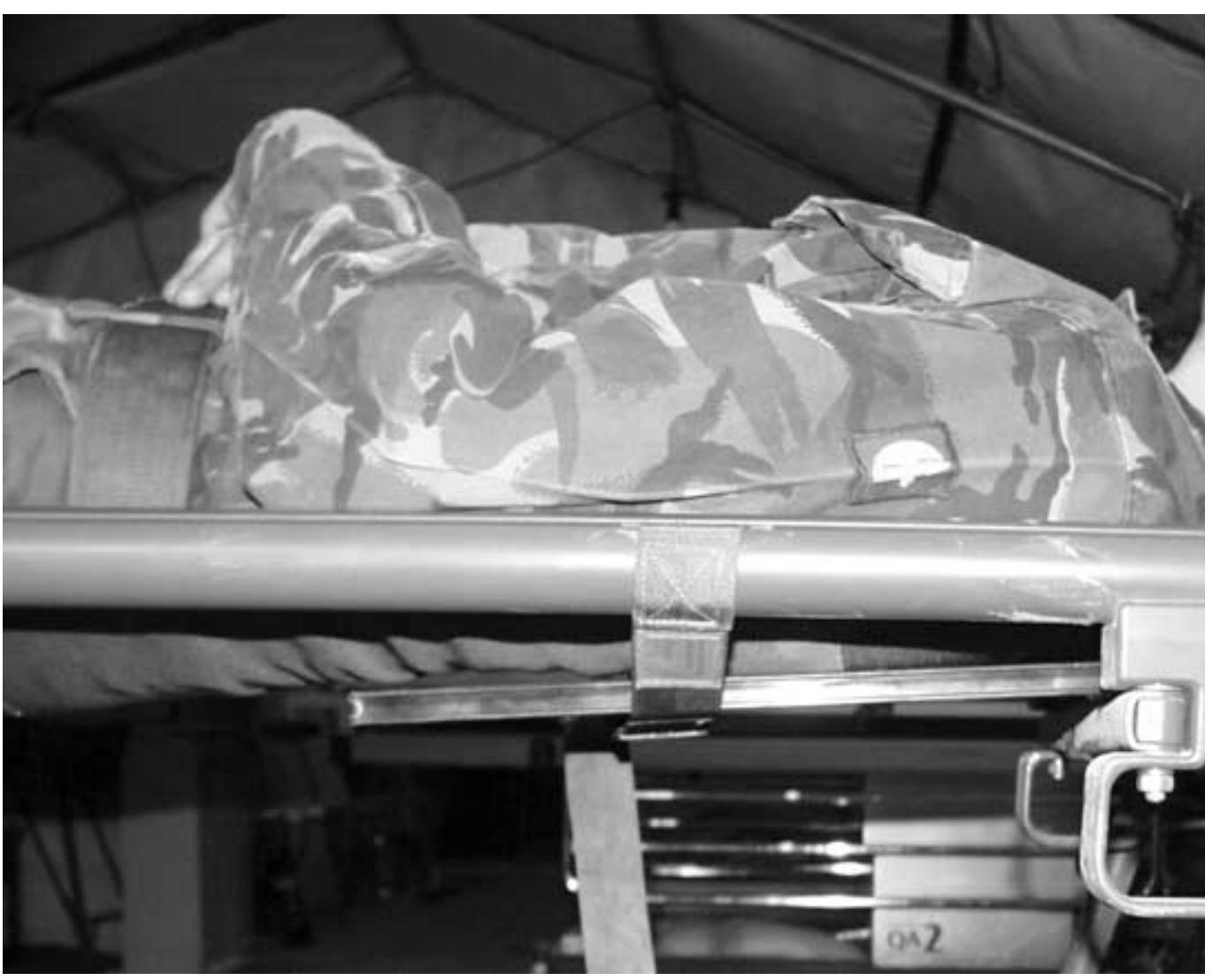

Fig 3. trestle position for chest radiograph. 